\title{
3. Insuficiencia cardiaca
}

\subsection{Miocarditis linfocítica. Abordaje diagnóstico de un caso}

Trujillo Virgen Erick Benjamín,

Polo Muñoz Manuel, Aceves García Moisés, Moreno Vázquez Alejandra, Becerril González Montecinos Alejandro, Lupercio Mora Karina Hospital de Cardiología del Centro Médico Nacional Siglo XXI. Tipo de estudio: Reporte de casos clínicos con revisión de fuentes

Introducción: La miocarditis es la inflamación del músculo cardiaco producida por una lesión isquémica, traumática, farmacológica o por agentes infecciosos. La causa más común es la infección viral por parvovirus B-19, HSV-6, adenovirus y enterovirus. Se reconoce que es la tercera causa de muerte súbita seguida de miocardiopatía hipertrófica y la enfermedad aterosclerótica. Puede haber daño miocárdico irreversible. Descripción del caso: Hombre de 44 años sin antecedentes crónico degenerativos. Presenta disnea de esfuerzo progresiva de 11 meses de evolución. Se inicia abordaje diagnóstico. Ecocardiograma transtorácico reporta dilatación ventricular izquierda, FEVI 15\%, SLG $3.22 \%$ TAPSE $12 \mathrm{~mm}$, onda S $8 \mathrm{~m} / \mathrm{s}$. Resonancia magnética: reforzamiento tardío no isquémico y datos de inflamación en región septal. Estudio histopatológico concluye miocarditis linfocítica activa con zonas de fibrosis leve a moderada. Metodología: A través del método booleano se realizó búsqueda de términos MeSH viral miocarditis, miocardiopatía inflamatoria, biopsia endomiocárdica y miocarditis en las bases de datos con repositorios de evidencia científica arbitrada indexada (PubMed) y no indexada (Google Scholar). Resultados: La miocarditis es una entidad clínica con una historia natural variable, puede ir de asintomática hasta choque cardiogénico, es importante reconocerla de forma rápida, ya que el inicio del tratamiento cambia la historia natural y puede limitar el daño miocárdico. En el ámbito

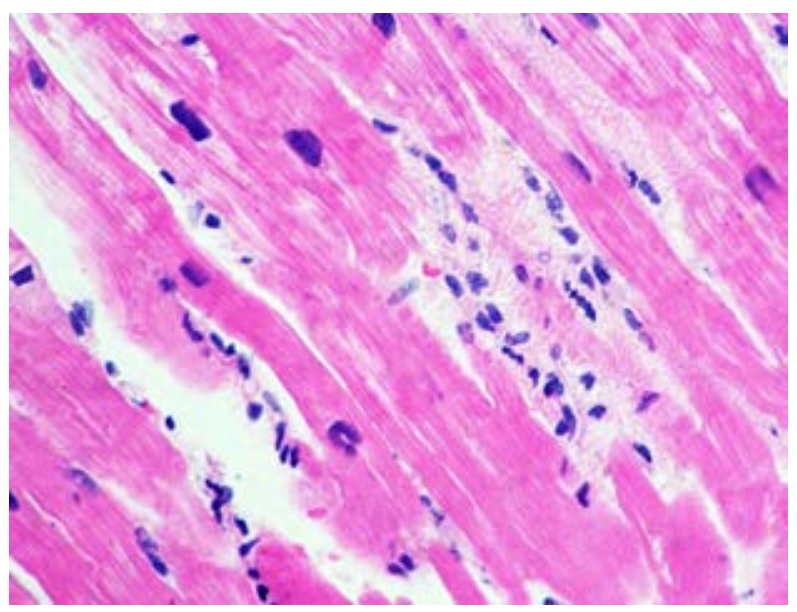

Figura 3.1.1: Biopsia endomiocárdica muestra un infiltrado inflamatorio por linfocitos asociado con daño de miocitos correspondiente a miocarditis linfocítica activa (H\&E 40×).
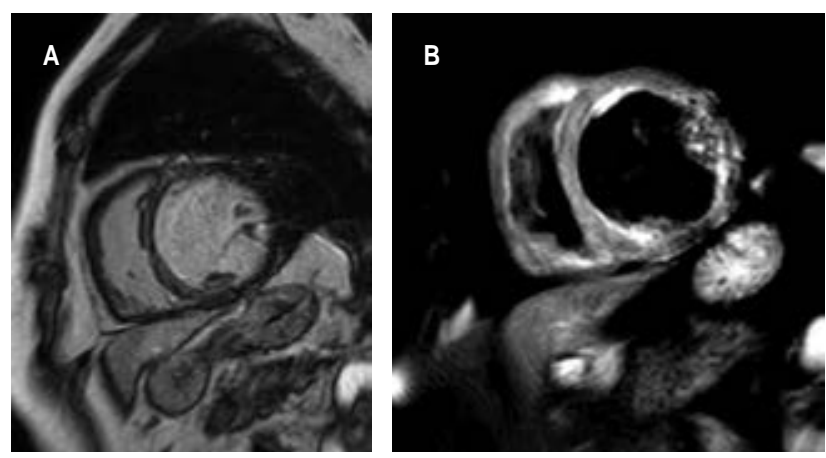

Figura 3.1.2: A) Secuencia de inversión de recuperación, reforzamiento intramiocárdico tardío del septum. B) T2 pesado con saturación grasa, edema intramiocárdico en septum.

mundial, la incidencia es de 22:100,000 año, con mayor afección al sexo masculino, edad media de 42 años. Discusión: La incidencia de miocarditis linfocítica es variable por regiones y epigenética de las distintas poblaciones, además el diagnóstico requiere de procedimiento invasivo y PCR. Los reportes de biopsias post mortem muestran una incidencia de $0.11 \%$. En este caso, el reconocerla oportunamente y realizar biopsia endomiocárdica permitió el tratamiento adecuado y mejorar el pronóstico.

\subsection{Cardiomiopatía diabética: abordaje diagnóstico de un caso}

Trujillo Virgen Erick Benjamín, Rodríguez Santiago Ramón Alejandro, Rayo Chávez Jorge, Olmos Domínguez Luis, Jiménez Santos Moisés, Almeida Gutiérrez Eduardo Hospital de Cardiología del Centro Médico Nacional Siglo XXI. Tipo de estudio: Reporte de casos clínicos con revisión de fuentes

Introducción: La falla cardiaca y la diabetes mellitus están íntimamente relacionadas, los resultados clínicos son considerablemente peores en pacientes con diabetes. A pesar de esta relación, no existe una definición universal para «cardiomiopatía diabética». En ausencia de un acuerdo, no es posible diagnosticarla clínicamente, por lo que se requiere de apoyo mediante alteraciones demostradas por estudios de gabinete. Descripción del caso: Mujer de 64 años, diabetes mellitus 2 de 14 años de evolución. Con disnea y angina a los medianos esfuerzos; mediante ecocardiograma transtorácico documentan FEVI 16\%, hipocinesia generalizada con dilatación del ventrículo izquierdo, coronariografía sin lesiones obstructivas. Se realizó resonancia magnética que expuso miocardiopatía dilatada no isquémica con incremento del volumen extracelular difuso en relación con cardiomiopatía diabética. Metodología: A través de método booleano se realizó búsqueda de términos $\mathrm{MeSH}$ diabetic cardiomyopathy, heart failure [and] diabetes mellitus en las bases de datos con repositorios de evidencia científica arbitrada indizada (PubMed) y no indizadas (Google Scholar). Resultados: El diagnóstico de la cardiomiopatía diabética es prácticamente por exclusión 
Figura 3.2.1:

Imagen por resonancia magnética nuclear, mapeo en

T1 tercio medio del ventrículo izquierdo. Reporta volumen extracelular de $35.8 \%$.

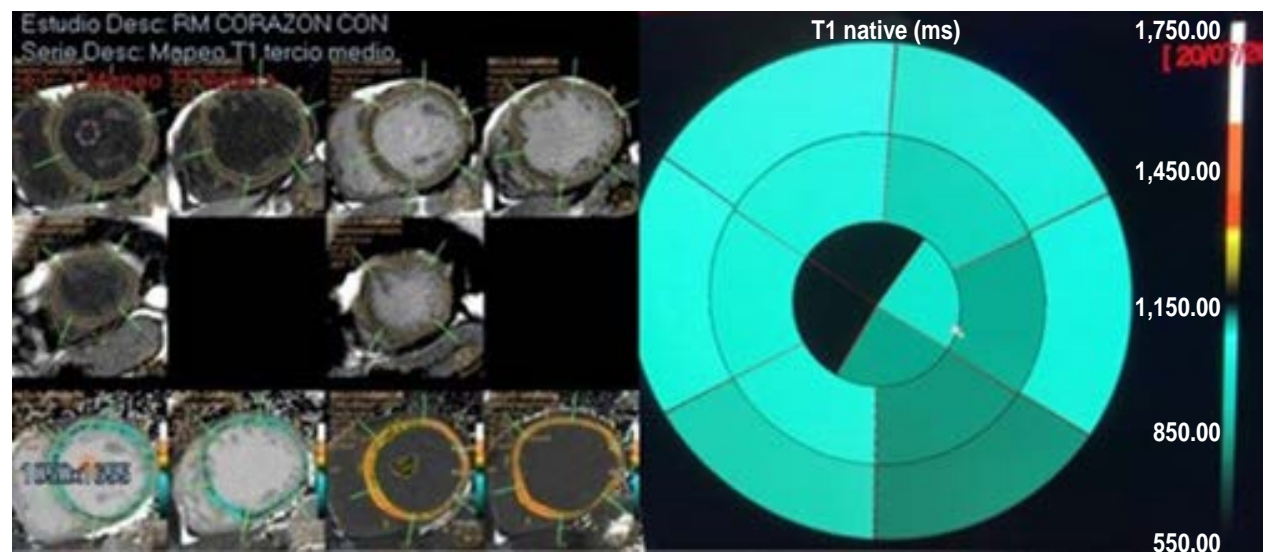

T1 nativo: regional result-1501, slice no: 2

\begin{tabular}{|c|c|c|c|c|c|c|c|}
\hline & Segmento 1 & Segmento 2 & Segmento 3 & Segmento 4 & Segmento 5 & Segmento 6 & Resumen \\
\hline T1 nativo & $1,052 \pm 59$ & $1,073 \pm 44$ & $1,075 \pm 60$ & $1,104 \pm 59$ & $1,102 \pm 204$ & $984 \pm 84.6$ & $1,066 \pm 106$ \\
\hline R1 nativo & $0.95 \pm 0.05$ & $0.93 \pm 0.04$ & $0.93 \pm 0.05$ & $0.91 \pm 0.05$ & $0.93 \pm 0.11$ & $1.02 \pm 0.10$ & $0.95 \pm 0.08$ \\
\hline T1 mejorado & $541 \pm 30.8$ & $552 \pm 26.1$ & $566 \pm 24.8$ & $503 \pm 38.7$ & $546 \pm 47.1$ & $546 \pm 33.8$ & $547 \pm 37.6$ \\
\hline R1 mejorado & $1.85 \pm 0.11$ & $1.82 \pm 0.09$ & $1.77 \pm 0.08$ & $2.00 \pm 0.16$ & $1.85 \pm 0.16$ & $1.84 \pm 0.11$ & $1.84 \pm 0.13$ \\
\hline$\varepsilon$ & $34.2 \%$ & $33.6 \%$ & $31.8 \%$ & $41.2 \%$ & $35.2 \%$ & $31.1 \%$ & $33.9 \%$ \\
\hline ROI área nativa & $163 \mathrm{~mm}^{2}$ & $165 \mathrm{~mm}^{2}$ & $257 \mathrm{~mm}^{2}$ & $187 \mathrm{~mm}^{2}$ & $169 \mathrm{~mm}^{2}$ & $169 \mathrm{~mm}^{2}$ & $1,110 \mathrm{~mm}^{2}$ \\
\hline ROI área mejorada & $148 \mathrm{~mm}^{2}$ & $174 \mathrm{~mm}^{2}$ & $280 \mathrm{~mm}^{2}$ & $115 \mathrm{~mm}^{2}$ & $154 \mathrm{~mm}^{2}$ & $162 \mathrm{~mm}^{2}$ & $1,034 \mathrm{~mm}^{2}$ \\
\hline Hematocrito & $45.0 \%$ & $45.0 \%$ & $45.0 \%$ & $45.0 \%$ & $45.0 \%$ & $45.0 \%$ & $45.0 \%$ \\
\hline Campo de fuerza & $1.5 \mathrm{~T}$ & $1.5 \mathrm{~T}$ & $1.5 \mathrm{~T}$ & $1.5 \mathrm{~T}$ & $1.5 \mathrm{~T}$ & $1.5 \mathrm{~T}$ & $1.5 \mathrm{~T}$ \\
\hline
\end{tabular}
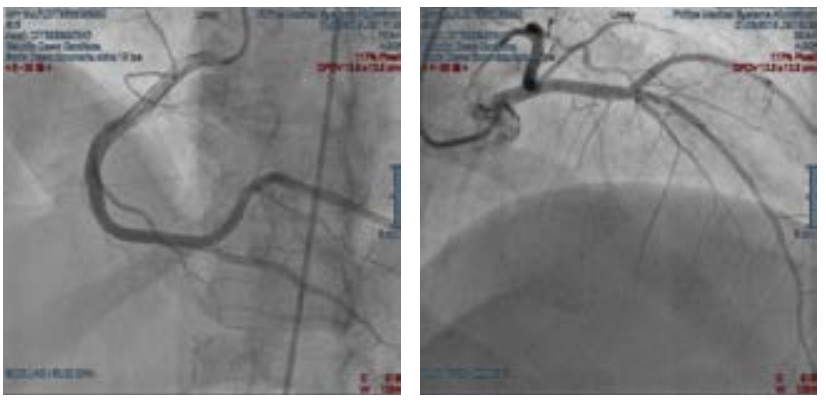

Figura 3.2.2: Coronariografía. Arterias coronarias epicárdicas sin lesiones angiográficamente significativas, flujo TIMI 3.

de otras causas de cardiomiopatía (isquémica, infiltrativa, etcétera), por ello, es fundamental el uso de estudios paraclínicos como la resonancia magnética para el correcto diagnóstico. Discusión: A pesar de la íntima relación entre la diabetes mellitus y la falla cardiaca, no existe un método diagnóstico ni tratamiento específico para la cardiomiopatía diabética, por lo que la conducta terapéutica está basada en la prevención y en la evidencia reunida en las guías actuales de falla cardiaca.

\subsection{Factores predictores de insuficiencia cardiaca en la diabetes tipo 2. Análisis del registro REMECAR}

Padilla Padilla Francisco Gerardo, ${ }^{*}$ Silva Giordano Alejandra, ${ }^{\ddagger}$ Pacheco Angélica, ${ }^{\S}$ García Luis ${ }^{\S}$
* Centro de Investigación Cardiología Clínica e Intervencionista. ${ }^{\ddagger}$ AstraZeneca. ${ }^{\S}$ ANCAM.

Tipo de estudio: Prueba diagnóstica

Introducción: La insuficiencia cardiaca es una complicación subdiagnosticada de manera temprana, sobre todo en los pacientes con diabetes mellitus tipo 2 (DMT2). Por lo que se requieren herramientas clínicas útiles para predecir la insuficiencia cardiaca e identificar a los casos con mayor riesgo. Algunos elementos clínicos de fácil determinación son muy útiles para realizar un diagnóstico temprano. Objetivo: Identificar los parámetros clínicos que pueden predecir la hospitalización por insuficiencia cardiaca según la escala TIMI, en la base del registro REMECAR. Metodología: Mediante el registro de 9,478 pacientes en REMECAR, identificamos casos con DMT2, utilizamos parámetros para cálculo de riesgo en hospitalización por insuficiencia cardiaca descritos por Berg y colaboradores. La escala de riesgo TIMI incluye: historia de insuficiencia cardiaca, historia de enfermedad arterial coronaria, fibrilación auricular, tasa de filtrado glomerular menor de $60 \mathrm{~mL} / \mathrm{min}$ y tasa albúminacreatinina. Resultados: Se analizaron 2,610 pacientes con DMT2, sólo 211 (8.1\%) tuvieron diagnóstico de insuficiencia cardiaca, encontramos historia de enfermedad coronaria en 575 (22\%). Fibrilación auricular en 95 casos. Tasa de filtrado glomerular menor de $60 \mathrm{~mL} / \mathrm{min}$ la encontramos en 541 personas. La tasa albúmina-creatinina fue un parámetro poco reportado y lo encontramos alterado en 13 pacientes. La gran mayoría de los casos analizados tuvieron un riesgo bajo 49\% (133) o intermedio $33 \%$ (76), alto riesgo 7\% (18), muy alto riesgo 10\% (23). Discusión y conclusiones: El uso de herramientas clínicas sencillas permite la detección de pacientes que tienen un alto riesgo de insuficiencia cardiaca. 